\title{
Low cost digitalization of observation telescope by utilizing smartphone
}

\author{
Ahmad Sohib ${ }^{1, *}$, Niko Danusaid ${ }^{1}$, Astri Sawitri ${ }^{1}$, Bebeh Wahid Nuryadin ${ }^{1}$, and Rena Denya Agustina ${ }^{2}$ \\ ${ }^{1}$ UIN Sunan Gunung Djati, Physics Department, Jln. A.H. Nasution No 106 Cibiru, Bandung 40614, Indonesia \\ ${ }^{2}$ UIN Sunan Gunung Djati, Physics Education Department, Jln. A.H. Nasution No 106 Cibiru, Bandung 40614, Indonesia
}

\begin{abstract}
Digitalization of telescopes used as learning media observation of the object is quite effective. However, the cost of operating this system becomes one of the obstacles. The approach to overcome the obstacle can be conducted by utilization of the present technology such as smartphone. Physical experiments have been conducted on the design of digitalization of the starter binoculars interfacing to Personal Computer (PC) using a smartphone. This experiment is aimed to design a more efficient digitalization of telescope observations. Smartphone stative is made in such a way that the camera in the right position on the telescope lens. Data retrieval is taken by a smartphone camera and ASI120MC camera as a comparison parameter. The data will be sent to the PC via an application installed both on smartphone and PC attributed by Bluetooth network. The camera is supported with a camera stative to keep it apart from binoculars. The observations obtained from this system is an interpretation between the camera on the telescope and PC. Such interpretations may produce images or videos observed by telescopes. This design can simplify the interfacing of telescope resulting good enough photo quality.
\end{abstract}

\section{Introduction}

Telescopes are devices that can widely be used to investigate an object much closer. Basically the telescope works by collecting the reflected light, then enlarged by the intern lens of the telescope, so the objects being investigated look much closer. Telescope can be used as a learning media in the field of astronomy recognition, technology development, or as a media of learning in other fields. Telescope, called Zadko telescope, was considered as the resource for enlarging and appealing interest in science education, especially astronomy in Australia. This kind of telescope is attributed by robotic controller and interface based webscheduling to allow controlling image observation and downloading data anywhere facilitated by speed internet [1].Today's technology is also developing a sophisticated use of telescopes. For instance, telescope observations are digitized by the help of software and conventional cameras or webcam [2] set on a telescope. The results of the observations can be analysed directly on the computer. But conventional cameras are still quite expensive, and it is rare for simple laboratory to have. Therefore, a low cost camera is required and has an excellent similarity to the conventional camera.

The most familiar and popular camera is the digital camera found in almost everyone's pocket. Over the last few years, there has been the development of many smartphone applications in various fields of research and education [3] [4]. Some of smartphone applications among the Microscopy is well-represented with polarized-light [5], Searching for ultra-high energy cosmic rays [6], biosensors [7], photo plethysmography [8], imaging and reading cholesterol test strips [9], fluorescence spectroscopy $[10,11]$, bioluminescence and other low-light applications [12], fluorometry for $\mathrm{pH}$ measurement in the field [13], and chemiluminescence [14]. Zeng, et al, also tried to construct a counting cell microscope based smartphone. The experiment result a low cost and portable smartphone microscope for biomedical and laboratory instrumentation application [15]. Moreover, in material physics, smartphones are also utilized to analyse particle sizes with an incorporation of lasers to replace dynamic light scattering (DLS) [16].

This paper is trying to make a minimalist approach to digitalization of telescopes in astronomy learning in the laboratory by utilizing smartphone. So that only with a smartphone camera and a computer featured a Bluetooth, telescope digitalization can be conducted easily and cheaply.

\section{Experiment}

\subsection{Smartphone}

The camera which was used in this experiment is Samsung Galaxy J2 Prime. The operating system of the smartphone is Android 6.0.1 Marshmallow and The internal RAM of the smartphone is $1.5 \mathrm{~GB}$. The smartphone also has resolution $540 \times 960$ pixels for

\footnotetext{
Corresponding author: asohib@,outlook.com
} 
display, approximately 220 ppi pixel density and $3264 \mathrm{x}$ 2448 pixel for camera. In addition, it is featured by Bluetooth 4.0 version.

\subsection{Application}

The application used to help this digitizing system is Smartcam. This open source app is installed both on smartphones and PCs. On the smartphone, this application in addition to acting as a picture taker as well as a transmitter, while Smartcam on PC acts as a receiver. The observed data will be sent via Smartcam on android through Bluetooth connection to the receiver on the PC.

\subsection{Accessories}

The accessories employed as a complement is a stativ as a smartphone holder on the telescope. This accessories is Stativ in this experiment is designed as in Fig. 1
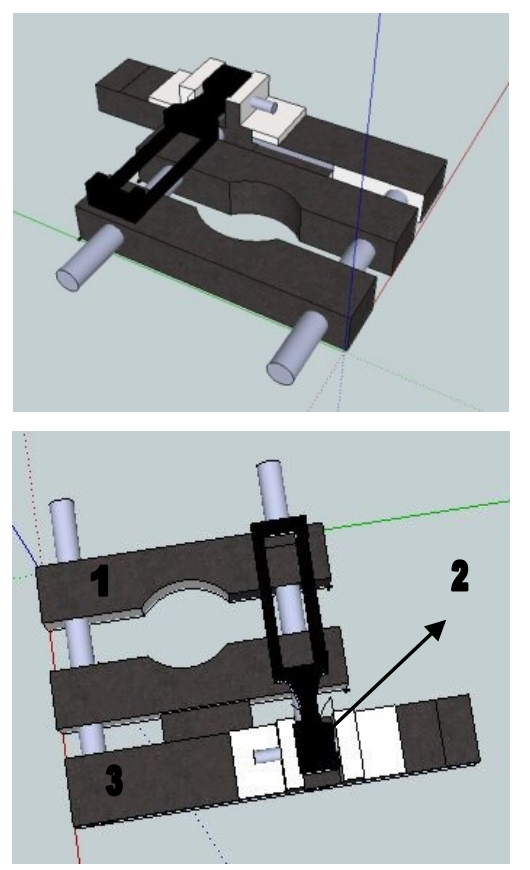

Fig. 1. Hand-made design of camera stative on the telescope.

The Stativ consists of three main components, (1) wood holder of lens, (2) holder of smartphone, and (3) wood base of smartphone holder. They were prepared by hand with dimensions $10 \mathrm{~cm} \times 2.5 \mathrm{~cm} \times 2.5 \mathrm{~cm}$ for (1) and $15 \mathrm{~cm} \times 2.5 \mathrm{~cm} \times 2.5 \mathrm{~cm}$ for (3). The wood holder of lens is shaped by semicircle form in the middle of the wood that serves for the telescope lens. Two nuts (20 $\mathrm{mm}$ diameter) were used to stick the parts. The Commercial smartphone holder (3) are housed on the base so that the smartphone can be right in the middle of the telescope lens.

\subsection{Snapshot Acquisition}

The acquisition of the snapshot is schematically shown in Fig. 2
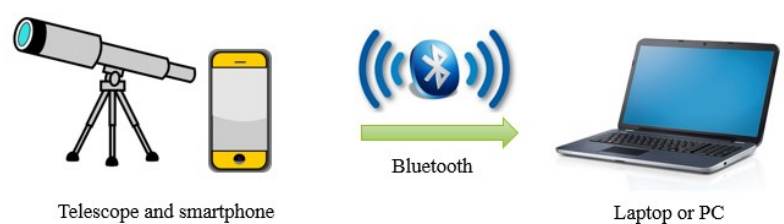

Telescope and smartphone

Fig. 2. Digitalization system schema of snapshotting acquisition.

The telescope (Celestron Brand) is directed to a leaf on a tree 10 meters from the observation room. The shooting is carried out employing a smartphone camera set above the telescope lens and connected to the PC using Bluetooth. The image obtained from this experiment will be compared with Image results with a webcam, ASI120MC, and Image taken in Bandung on March 16 $6^{\text {th }}, 2016$ (Sun Eclipse moment) by DLSR camera.

\section{Result}

This experiment resulted by a smartphone-based digitalization design of the object observation employing a telescope and it is shown by Fig. 3. The Fig. 3 (a) show the system utilized smartphone as the camera of the system has not been connected to the PC,

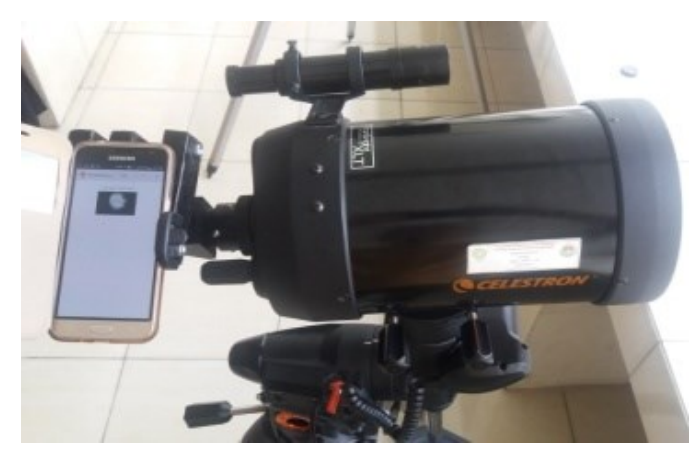

(a)

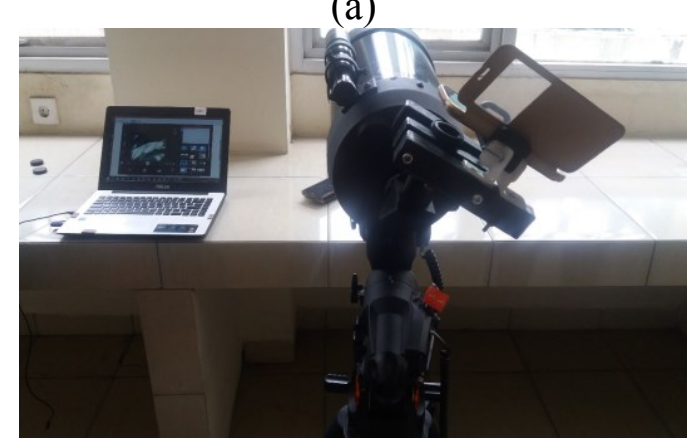

(b)

Fig. 3. Digitalization design (a) before and (b) after connected to PC. 
but Fig.3 (b) shows that the system has been ready to employ. The system seems more flexible and simple than that of what was conducted by Ayu, et al [2].

Fig. 4 shows the images resulted by the smartphonebased digitalization system. The images were captured by the low magnitude Fig. 4. (a), four times larger magnitude (b), and ASI120MC (c) in telescope observations. In Fig. 4 (a) the observed object is surrounded by black shadow, which is a lens casing on the telescope. The casing is observed because there is a distance between the camera lens and the telescope lens. So at the minimum magnification, the lens casing will be observed. However in the Fig. 4(b), the casing lens is

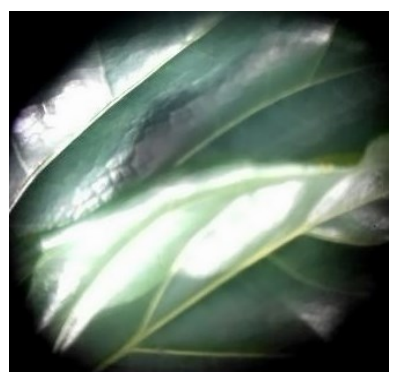

(a)

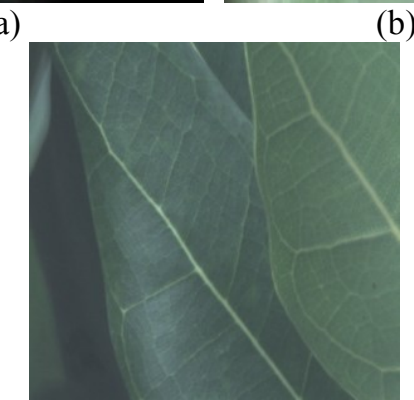

(c)

Fig. 4. Pictures captured by (a) smartphone with low magnitude, (b) smartphone with larger magnitude, and (c) Commercial camera ASI120MC.

Not captured anymore, due to its larger magnification. Nevertheless the Fig. 4 (c) shows the observed object clearly.

It is obvious that both of the images produced by smartphone-based system has high lighting. The sheen attributed on the images is caused by the reflection of the outer light from the telescope lens to the camera lens. The reflected light pollution is due to the existence of a distance between the camera lens of the smartphone and the telescope lens without any covering, so it makes the light easy to catch. In contrary, the image captured by employing a webcam appears more obviously. This is caused the webcam directly observe the object on the mirror taken place in the telescope. There is no light pollution coming in on the webcam lens. But the observations with smartphones on the maximum magnitude is almost similar to the observations with webcam.

The other object which was investigated by the system was the sun. the observation was conducted in the astronomy laboratory at $10 \mathrm{am}$. The Fig. 5 is snapshot of the sun obtained by the system. Sun pictures captured by smartphone with low magnitude, smartphone with four times larger magnitude, and low magnitude DSLR camera are respectively shown by Fig. 5 (a), (b), and (c).

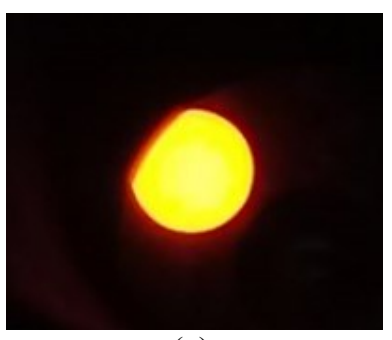

(a)

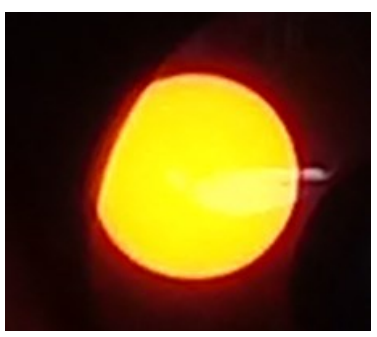

(b)

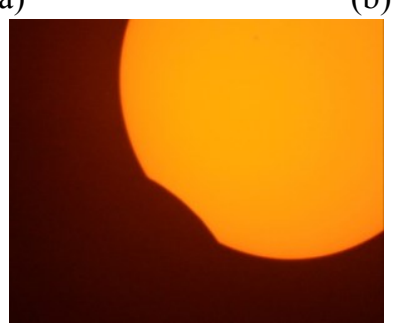

(c)

Fig. 5. Pictures captured by (a) smartphone with low magnitude, (b) smartphone with larger magnitude, and (c) DSLR camera.

The objects shown in the picture (a) look quite clear, as well as the picture (b), although the sun observed by DSLR camera (c) is more obvious. The dark shadow in the images (a) and (b) that cover few parts of the objects and cause the observed sun to appear definitely. The shadow is created by the lens cover wall of the telescope. Nevertheless the shadow appears in the image (c) was the moon, because the image was obtained when the eclipse occurred in March 2016. The other hand, when the images have some diversities from their colors. The images produced by smartphone-based system are lighter than that of DSLR-based system. The color diversity of the images is led by the pixel size of the camera that can affect the saturation of the image.

As it is tried to be calculated about the cost for smartphone -based digitalization system, of course, the cost required is much lower than that of the webcambased system. It can replace the webcam to be employed as a learning media telescope.

\section{Conclusion}

The smartphone-based digitalization design for object observations with telescopes was successfully created. Images obtained from this digitizing system can be displayed directly on the computer. With this camera's stative camera design, digitalization can improve the lower cost effectiveness and it is appropriate to be applied as a learning tool for telescope learning media.

The author personally wishes to thank to the members of PASCAL 2014 A, especially Cece Solihin, Haekal Pazha, Fakhrizal Muttaqien, Mira, and Indriani Rahmawati, for financial support and assistance. 


\section{References}

1. D.M. Coward, A. Heary, G. Venville, M. Todd, M. Laas-Bourez, M. Zadnik, A. Klotz, M. Boe“r, N. Longnecker, Adv. Space Research 47, 1922 (2011)

2. R. Ayu, R. Priyatikanto, E. Soegiartini, H.L. Malasan, and M. Arifyanto, Proc. Indones. Astron. Ascossiation (2003)

3. E. Novitasari, Supurwoko, and Surantoro, Pendidik. Fis., 1, 37 (2013)

4. R. Twum, J. Edu. Prac. 8, 216 (2017)

5. C.W. Pirnstill, G.L. Coté, Sci. Rep. 5, 13368 (2015)

6. D. Whiteson, M. Mulhearn, C. Shimmin , K. Cranmer, K. Brodie, D. Burns, Astroparticle Phy. 79, 1 (2016)

7. D. Zhang, Q. Liu, Biosens Bioelectron 75, 273 (2016)

8. S. Kwon, H. Kim, K.S. Park, Proc. IEEE Annual International Conference on Engineering and Medicine and Biology, 2174 (2012)

9. V. Oncescu, M. Mancuso, D. Erickson, Lab on a Chip, 14, 759 (2014)

10. M.A. Hossain, J. Canning, S. Ast, K. Cook, P.J. Rutledge, A. Jamalipour, Opt. Lett. 40, 1737 (2015)

11. H.J. Yu, Y.F. Tan, B.T. Cunningham, Anal. Chem. 86, 8805 (2014)

12. H. Kim, Y. Jung, I.J. Doh, R.A. Lozano-Mahecha, B. Applegate, E. Bae . Sci. Rep.7 40203 (2017)

13. M.A. Hossain , J. Canning, S. Ast, P.J. Rutledge, T.L. Yen, A. Jamalipour, IEEE Sens. J. 15, 5095 (2015)

14. M. Zangheri, L. Cevenini, L. Anfossi, C. Baggiani, P. Simoni , F. Di Nardo , Biosens Bioelectron, 64, 63 (2015)

15. Y. Zeng, K. Jin, J. Li, J. Liu, J. Li, T. Li, S. Li, Sensors and Actuators (to be published)

16. S. Nasution, Developing of Dynamic Light Scattering as Particle Size Analyzer by Camera (Thesis, Bandung, 2018) 